\title{
A New Pinane-Type Tridentate Modifier for Asymmetric Reduction of Ketones with Lithium Aluminum Hydride
}

\author{
Yie-Jia Cherng ${ }^{a}$, Jim-Min Fang, ${ }^{a, *}$ and Ta-Jung Lu ${ }^{b, *}$ \\ a Department of Chemistry, National Taiwan University, Taipei, Taiwan, 106 R.O.C. \\ b Department of Chemistry, National Chung-Hsing University, \\ Taichung, Taiwan, 402 R.O.C.
}

\begin{abstract}
The reduction of aryl and alkenyl methyl ketones using lithium aluminum hydride modified with $(1 R, 2 S, 3 S, 5 R)-(+)$-10-anilino-3-ethoxy-2hydroxypinane (3), afforded chiral secondary alcohols in 83-96\% yields and 53$97 \%$ optical yields. The modifier 3 was prepared from $(1 R)$-(-)-myrtenol and was readily recovered $(>96 \%)$ after reduction. The reduction of acetophenone in the presence of lithium iodide gave the alcohol product with higher optical yield.
\end{abstract}

Enantiomerically pure chiral secondary alcohols are important starting materials for the total synthesis of natural products. ${ }^{1}$ High degrees of asymmetric induction have been realized in the reduction of prochiral ketones using chirally modified lithium aluminum hydride (LAH) reagents to give optically active secondary alcohols. ${ }^{2}$ We have recently demonstrated that secondary alcohols can be obtained in moderate optical yields in the reduction of prochiral ketones by LAH using pinane-based modifiers 1 and $2^{3}$

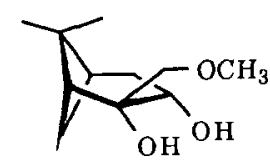

1

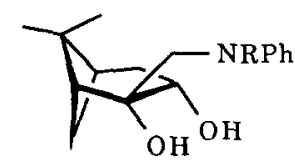

2a, $\mathrm{R}=\mathrm{H} ; \mathbf{2 b}, \mathrm{R}=\mathrm{CH}_{3}$

After molecular modelling analysis of the putative transition structures ${ }^{3}$ in an attempt to develop more effective modifiers, we envisaged that a C-3 alkoxy group is sterically more demanding than a hydroxyl group and therefore can better differentiate the two reaction faces of the reactant. Thus a new, rationally designed, pinane-based modifier 3 , possessing a rigid bicyclic pinane skeleton and an ethoxy substituent at $\mathrm{C}-3$, has been prepared. Compound 3 proved to be very efficient in the sense of product yields, optical yields and reuse of the modifier. We herein describe the preparation of $(1 R, 2 S, 3 S, 5 R)-(+)-10$-anilino-3-ethoxy- 
2-hydroxypinane (3) from (1R)-(-)-myrtenol and its application as an improved chiral modifier for lithium aluminum hydride in the reduction of prochiral ketones.

As delineated in Scheme I, $(1 R)-(-)$-myrtenol was reacted with $\mathrm{PBr}_{3}$ to give $(1 R)-(-)-$ myrtenyl bromide in $85 \%$ yield ${ }^{4}$ which was converted to the desired diol $2 \mathrm{a}$ by the known procedure. $^{3}$ Selective alkylation of the secondary hydroxyl group with sodium hydride and bromoethane furnished the desired modifier 3 in $83 \%$ yield. $^{5}$

\section{Scheme I}

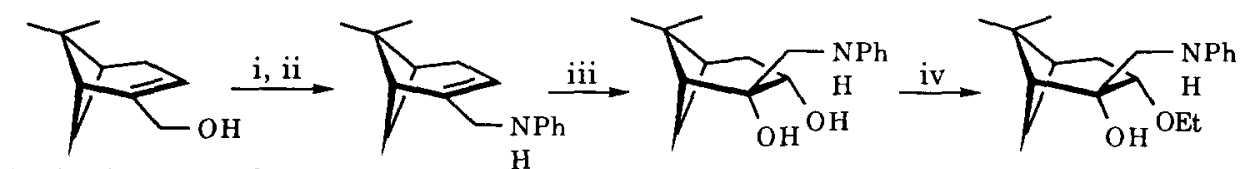

$(1 R)-(-)$-myrtenol

4

$2 a$ 3

i. $\mathrm{PBr}_{3}$ /pyridine/benzene, $\mathrm{rt}, 85 \%$

ii. $\mathrm{KH} /$ aniline/THF, $\mathrm{rt}, 85 \%$

iii. $\mathrm{OsO}_{4} /\left(\mathrm{CH}_{3}\right)_{3} \mathrm{~N} \rightarrow \mathrm{O}$, reflux, 50\%

iv. $\mathrm{NaH} / \mathrm{EtBr}, 83 \%$

The chiral reducing agent was prepared in situ by mixing a standardized stock solution of LAH in ether with modifier 3 at room temperature for $1 h^{3,6}$ THF solutions of prochiral ketones were then added at $-78^{\circ} \mathrm{C}$ and stirred for $60 \mathrm{~min}$ to furnish the corresponding secondary alcohols in $83-96 \%$ chemical yields (Table 1). The chiral modifier 3 was recovered nearly quantitatively $(>96 \%$ ) from the reaction mixture by silica-gel chromatography. It was noted in the reduction of acetophenone and 4-phenyl-3-buten-2-one that the enantioselectivities varied remarkably in different solvent systems. ${ }^{7}$ A 1:10 mixture of $\mathrm{Et}_{2} \mathrm{O} / \mathrm{THF}$ appeared to be the solvent of choice for the asymmetric LAH reduction. In such a solvent system, several mono- and disubstituted acetophenones were reduced by $\mathrm{LAH} /$ modifier 3 to afford the corresponding phenethanols in favor of the $(R)$-configuration (66-97\% optical yields). The reduction of methyl 2-nitrophenyl ketone (entry 7) was not prevented by the nitro group and gave $85 \%$ of the desired alcohol. 2-Acetylfuran, 1acetylnaphthalene, 2-acetylnaphthalene, 2-acetylphenanthrene and 2-acetylfluorene were similarly reduced to give $55,85,53,93$ and $86 \%$ optical yields respectively. As the addition of HMPA or lithium iodide is known to affect the enantioselectivities in certain reactions (such as alkylation reactions of enolates or allylmetals), ${ }^{8}$ we also examined their effects in the LAH reductions. The preliminary results indicated that the optical yield of the alcohol products were increased in some instances by addition of LiI (entries 2,12 , and 18). On the other 
hand, the enantioselectivity decreased in the reduction of 1-acetylnaphthalene in the presence of the cosolvent HMPA (entry 13).

Table 1 Reduction of methyl ketones $\mathrm{RCOCH}_{3}$ with $\mathrm{LiAlH}_{4}$ and chiral modifier 3 . $^{\mathrm{a}}$

\begin{tabular}{|c|c|c|c|c|c|c|}
\hline entry & Ar & $\begin{array}{l}\text { additive } \\
\text { (equiv) }^{b}\end{array}$ & $\begin{array}{c}\text { product } \\
\text { yield }(\%)^{\mathrm{c}}\end{array}$ & $\begin{array}{c}\text { ee } \\
(\%)^{\mathrm{d}}\end{array}$ & $\begin{array}{c}\text { optical yield } \\
(\%)^{\mathrm{e}}\end{array}$ & config. \\
\hline 1 & $\mathrm{Ph}$ & & 85 & 62 & 66 & $R$ \\
\hline 2 & $\mathrm{Ph}$ & LiI (1) & 91 & 83 & 88 & $R$ \\
\hline 3 & o-tolyl & & 86 & 79 & 84 & $R$ \\
\hline 4 & 2-bromophenyl & & 86 & 78 & 83 & $R$ \\
\hline 5 & 2-chlorophenyl & & 86 & 78 & 83 & $R$ \\
\hline 6 & 2-methoxyphenyl & & 86 & 91 & 97 & $R$ \\
\hline 7 & 2-nitrophenyl & & 85 & 72 & 77 & $R$ \\
\hline 8 & 2,4-dimethylphenyl & & 87 & 74 & 79 & $R$ \\
\hline 9 & 2,5-dimethoxyphenyl & & 91 & 91 & 97 & $R$ \\
\hline 10 & 2-furyl & & 88 & 52 & 55 & $R$ \\
\hline 11 & 1-naphthyl & & 93 & 80 & 85 & $R$ \\
\hline 12 & 1-naphthyl & LiI (1) & 92 & 84 & 90 & $R$ \\
\hline 13 & 1-naphthyl & HMPA (6) & 85 & 66 & 70 & $R$ \\
\hline 14 & 2-naphthyl & & 96 & 50 & 53 & $R$ \\
\hline 15 & 2-phenanthyl & & 94 & 87 & 93 & \\
\hline 16 & 2-fluorenyl & & 95 & 81 & 86 & \\
\hline 17 & $\mathrm{PhCH}=\mathrm{CH}$ & & 93 & 50 & 53 & $R$ \\
\hline 18 & $\mathrm{PhCH}=\mathrm{CH}$ & $\operatorname{LiI}(1)$ & 91 & 52 & 55 & $R$ \\
\hline 19 & 1-cyclohexenyl & & 89 & 74 & 79 & $R$ \\
\hline
\end{tabular}

a. The reactions were carried out in ether/THF (1:10) solution with the following stoichiometry: LAH/modifier 3/ketone $=2 / 2.2 / 1$. b. Based on ketone. c. Isolated yields. $d$. The optical yield is determined by HPLC analysis ${ }^{9}$ the major enantiomer has the $(R)$-configuration. e. Calculated based on the maximum optical purity (93.8\%) of the modifier 3 .

A wide range of aromatic and alkenyl methyl ketones can be reduced by LAH in the presence of modifier 3 to give chiral secondary alcohols in synthetically useful yields and enantioselectivities. The facial selectivities realized in the LAH reduction of aromatic methyl ketones using modifier 3 are superior to those utilizing simple terpenic diol modifiers. ${ }^{10}$ The reduction of alkenyl methyl ketones (entries 17-19) gave exclusively the allylic alcohols, no 1,4-reduction products were observed. Our results demonstrate the importance of the amino substituent at C-10 in modifier 3 for the chiral induction in the asymmetric reduction of 
ketones using chiral LAH reagents. The asymmetric induction was further improved by the ethoxy group at C-3 of modifier 3 compared with the hydroxyl group in modifiers 1 or 2.

Acknowledgments We thank the National Science Council of the Republic of China (NSC82-0208-M-005-021 \& NSC82-0208-M-002-031) for generous financial support of our research programs and to the regional instruments centers of NSC for sample analyses.

\section{References and Notes}

1. (a) Noyori, R.; Tomino, I.; Nishizawa, M. J. Am. Chem. Soc. 1979, 101, 5843. (b) Grigg, R.; Santhakumar, V.; Sridharan, V.; Thornton-Pett, M.; Bridge, A. W. Tetrahedron $1993,49,5177$.

2. For recent reviews on reducing agents, see: (a) Midland, M. M. Chem. Rev. 1989, 89, 1553. (b) Singh, V. K. Synthesis 1992, 605 and the references cited therein.

3. (a) Lu, T.-J.; Liu, S.-W. J. Chin. Chem. Soc. 1994, 41, 205. (b) Lu, T.-J.; Liu, S.-W. J. Chin. Chem. Soc. 1994, 41, 467.

4. (a) For converting an alcohol to a bromide, see: Noller, C. R.; Dinsmore, R. Org. Synth. Coll. Vol. 2, 1943, 358. (b) For the preparation of myrtenyl bromide from $\beta$-pinene, see: Zweifel, G.; Whitney, C. C. J. Org. Chem. 1966, 31, 4178.

5. Modifier 3 gave satisfactory elemental analysis and was converted to $(1 R, 2 S, 3 S, 5 R)-(+)-$ 10-(3,5-dinitrobenzanilido)-3-ethoxy-2-hydroxypinane (5) by reacting it with 3,5-dinitro benzoyl chloride in the presence of triethylamine in THF. The optical purity of the corresponding 3,5-dinitrobenzamide 5 was determined to be $93.8 \%$ ee by HPLC analysis using a CHIRALCEL OD (Daicel Chemical Ind. Ltd.) column (25 cm x $4.6 \mathrm{~mm}$ ), eluted with 2-propanol/hexane $(1 / 20$, flow rate: $0.42 \mathrm{~mL} / \mathrm{min})$. The retention times for the major isomer [(+)-5] is $31.94 \mathrm{~min}$ and the minor is $42.78 \mathrm{~min}$.

6. (a) Landor, S. R.; Miller, B. J.; Tatchell, A. R. J. Chem. Soc. (C) 1966, 2280. (b) Landor, S. R.; Miller, B. J.; Tatchell, A. R. J. Chem. Soc. (C) 1967, 197. (c) Landor, S. R.; Miller, B. J.; Tatchell, A. R. J. Chem. Soc. (C) 1971, 2339. (d) Landor, S. R.; Sonola, O. O.; Tatchell, A. R. J. Chem. Soc., Perkin Trans. I 1978, 605. (e) Baggett, N.; Stribblehill, P. J. Chem. Soc., Perkin Trans. I 1977, 1123.

7. The enantiomeric excesses dropped dramatically when the reactions were carried out in nonpolar solvents such as diethyl ether.

8. (a) Seebach, D. Angew.Chem., Int. Ed. Engl. 1988, 27, 1624. (b) Chang, C.-J.; Fang, J.M.; Liao, L.-F. J. Org. Chem. 1993, 58, 1754 and the references cited therein.

9. The enantiomeric excess of the resulting alcohol was determined by HPLC analysis using a CHIRALCEL OD column, eluted with 2-propanol/hexane (1/500, flow rate: 1.0 $\mathrm{mL} / \mathrm{min}$ ).

10. Haller, R.; Schneider, H. J. Chem. Ber. 1973, 106, 1312. 